\title{
Las competencias laborales como variable vinculada al aprendizaje organizacional en instituciones educativas
}

\section{RESUMEN}

El propósito del presente estudio es analizar las relaciones que pueden existir entre las competencias laborales y el aprendizaje organizacional en docentes de instituciones educativas estatales del distrito de Comas. Se tomo una muestra de 240 docentes a quienes se les aplicó dos instrumentos de evaluación, uno para cada variable. Los resultados indican que existen correlaciones significativas y positivas entre las variables de estudio, igualmente se encontró que las docentes mujeres presentan mejores resultados en el aprendizaje organizacional que los docentes varones.

Palabras Claves: Competencias laborales, Aprendizaje organizacional, Calidad Académica, educación.

THE LABOUR COMPETITIONS

AS A VARIABLE LINKED TO

ORGANIZATIONAL LEARNING IN

TEACHERS

\section{ABSTRACT}

The purpose of the present study is analyzing the relations that could exist between the labour competitions and the organizational learning in teachers from Comas' public educational institutions. A sample of 240 teachers was taken and two evaluations instruments were applied, one for each variable. The results show that significant and positives correlations exist between the variables of study, furthermore it was found that women teachers have better results in the organizational learning than men teachers.

Keywords: Labour competitions, organizational learning, Academic quality, education

\section{FUNDAMENTACION TEORICA}

En la sociedad del conocimiento, los cambios acelerados que se producen son aspectos centrales de la revolución tecnológica que vivimos y que encuentran su mayor expresión en el campo de las comunicaciones informáticas. Pero el mayor aporte de esta revolución tecnológica, consiste en alentar el incremento de la oferta de valor-conocimiento creativo. Ese valor - conocimiento creativo, no es más que la generación de aprendizaje; es decir, adquirir, almacenar, diseminar, aplicar y utilizar conocimiento. La gestión del conocimiento procura maximizar el aprendizaje organizacional con miras a incrementar su competitividad global.

Durante los últimos años numerosos autores abundan en considerar al conocimiento como el recurso más importante que posee una organización para conseguir y mantener una ventaja competitiva (Drucker, 1993) (1). Se entiende aquí conocimiento como un fenómeno creado y acumulado a través de un proceso denominado aprendizaje organizacional (Pawlowsky 2001) (2).

El aprendizaje organizacional ocurre cuando "los individuos de la organización experimentan una situación problemática y se preguntan por el comportamiento de la organización"; por otra parte, "el aprendizaje, para que pueda ser considerado organizacional, debe ser incorporado mediante artefactos epistemológicos (mapas, memorias y programas) que se encuentren en el ambiente de la organización" (Argyris, 2002) (3).

Es por eso que el eficiente funcionamiento de cualquier tipo de organización requiere de planeación, desarrollo y evaluación. Eficiencia y eficacia, son dos términos fundamentales que obligan a cumplir con ciertos objetivos y hacerlo con el mínimo de recursos. Para lograrlo se necesita integrar acciones y reflexiones con conocimientos, habilidades, actitudes y recursos. En tal sentido, es importante cuidar las condiciones que favorecen la formación de comunidades de aprendizaje en donde se da de manera eficiente la gestión del conocimiento, haciendo posible que emerjan ambientes de aprendizaje que propicien la creación, circulación, tratamiento y utilización de dicho conocimiento. Aprender a aprender en estas circunstancias es vital en la sociedad del conocimiento, tal como son las demandas actuales en las que la calidad se ha convertido en el objetivo vital de cada organización.

\footnotetext{
Doctor. Docente Principal de la Facultad de Psicología UNMSM. E-mail: mtorresv@unmsm. edu.pe

2 Docente FIIS-UNI . E-mail: huamans@gmail.com
} 
En este contexto las universidades han desempeñado un papel muy importante en la formación profesional de nuestros jóvenes, tratando de satisfacer las necesidades que demanda la industria de bienes y servicios; no obstante, en la actualidad, debido al fenómeno de la globalización, es necesario conocer las necesidades de los mercados externos con el fin de dar una visión más amplia a los futuros profesionales, además de enfatizar la necesidad de dar un enfoque empresarial a cada carrera para producir más líderes y menos empleados.

En el mismo orden de ideas, las tendencias modernas obligan a ir creando profesionales con capacidades multidisciplinarias, es decir, hábiles para dirigir, administrar, generar y manejar técnicamente un grupo de trabajo y una empresa de cualquier ramo, especialidad y país a fin de lograr, en una primera instancia, ser autosuficientes económicamente, y a la vez ser precursores de empleos para otras personas en bien del país y de la economía global.

A pesar de las dificultades que esto acarrea, muchas universidades están cambiando su visión y sus alcances con deseos de lograr la excelencia académica a nivel nacional e internacional, y a la vez llevar la idea de liderazgo a todas sus esferas de acción. Para ello, se han planteado la tarea de convertirse en organizaciones que aprenden, han iniciado una labor de reingeniería en sus programas y han establecido una política de apertura al cambio y a la renovación constante en busca del éxito, no sólo de ellas como instituciones, sino de sus egresados como producto fehaciente y claro de su labor cumplida.

Ante un entorno que cada día exige mejorar, no tanto para ir a la vanguardia, sino tan sólo para mantenerse, surgen por todas partes los programas de mejora continua. Sin embargo, es un hecho que para que una organización mejore, primero tiene que aprender algo nuevo, porque de lo contrario, sólo se estarían repitiendo viejas prácticas y los cambios que se logren sólo serían superficiales y de corto plazo.

Por ello, es sumamente importante en la actualidad estar consciente de la necesidad de mantenerse en constante búsqueda del mejoramiento y actualización, debido a la rapidez con que se está moviendo la información en los tiempos modernos, así como no aferrarse a modelos educativos estáticos supuestamente terminados, si se quiere mantenerse vigente y eficiente. Por tanto, es primordial motivar al personal docente a continuar aprendiendo, a involucrarse en un proceso de investigación y perfeccionamiento constante, nunca acabado, para, con ello, dar un ejemplo evidente de lo que los estudian- tes tendrán que vivir haciendo: preparándose toda la vida, a fin de enfrentarse con más armas y estrategias (conocimientos y experiencias) a la constante competencia en el mercado laboral y profesional.

Pero esta tarea no puede quedarse solo en el nivel universitario. La velocidad con que se suceden los cambios debe llevar a que, necesariamente, las instituciones educativas de nivel básico puedan convertirse en organizaciones que aprenden de tal manera que los docentes y los alumnos puedan convertirse en aprendices estratégicos y con ello avanzar progresivamente hacía la consecución del objetivo final del proceso educativo: una educación de calidad.

A pesar de los avances logrados en los últimos tiempos, la realidad nos indica que hay mucho trabajo que realizar; los informes internacionales y nacionales sobre la evaluación de alumnos y docentes nos muestran una realidad lamentable; bajos niveles de comprensión lectora y razonamiento matemático por parte de los alumnos y un altísimo índice de profesores desaprobados. Estos hechos son muestras claras de que debemos redoblar esfuerzos para mejorar la calidad de la educación y una buena forma de hacerlo es convirtiendo a nuestras instituciones educativas en organizaciones que aprenden.

Dentro de este proceso el desempeño profesional de los docentes es uno de los elementos más importantes que debemos atender (Valdez, 2009) (4). La calidad docente puede entenderse como un objetivo a conseguir en tres etapas realimentadas: la confección del programa de la asignatura, la adopción de un enfoque metodológico y la evaluación de los resultados. Si bien se trata de un objetivo común a todo proceso de enseñanza, las formas de conseguirlo dependen del tipo de habilidades, aptitudes y conocimientos que se han de impartir y de los medios tecnológicos disponibles.

El propósito fundamental de la presente investigación es establecer las probables relaciones que pueden existir entre el aprendizaje organizacional y el desempeño profesional de los docentes de las instituciones educativas del distrito de Comas, con la finalidad de buscar alternativas viables que nos sirvan para mejorar la calidad del proceso educativo y adecuarlo a las exigencias que demanda el mundo de hoy.

Como consecuencia de lo expuesto, los problemas de la presente investigación se plantean de la siguiente manera:

- ¿Que relación existe entre las competencias laborales y el aprendizaje organizacional en 
los docentes de las instituciones educativas del distrito de Comas?

- ¿Cuáles son los niveles de aprendizaje organizacional que presentan los docentes de las instituciones educativas del distrito de Comas?

- ¿Cuáles son los niveles de competencias laborales que presentan los docentes de las instituciones educativas del distrito de Comas?

- ¿Qué diferencias existen respecto del aprendizaje organizacional entre los docentes varones y mujeres de las instituciones educativas del distrito de Comas?

- ¿Qué diferencias existen respecto de las competencias laborales entre los docentes varones y mujeres de las instituciones educativas del distrito de Comas?

\section{OBJETIVOS}

- Establecer las relaciones que existen entre las competencias laborales y el aprendizaje organizacional en los docentes de las instituciones educativas del distrito de Comas.

- Describir los niveles de aprendizaje organizacional que presentan los docentes de las instituciones educativas del distrito de Comas.

- Describir los niveles de competencias laborales que presentan los docentes de las instituciones educativas del distrito de Comas.

- Establecer las diferencias que existen respecto del aprendizaje organizacional entre los docentes varones y mujeres de las instituciones educativas del distrito de Comas.

- Establecer las diferencias que existen respecto de las competencias laborales entre los docentes varones y mujeres de las instituciones educativas del distrito de Comas.

\section{HIPÓTESIS}

- Existen relaciones significativas entre las competencias laborales y el aprendizaje organizacional en los docentes de las instituciones educativas del distrito de Comas.

- Existen diferencias significativas respecto del aprendizaje organizacional entre los docentes varones y mujeres de las instituciones educativas del distrito de Comas.

- Las competencias laborales difieren significativamente entre los docentes varones y mujeres de las instituciones educativas del distrito de Comas.

\section{MÉTODOS Y RESULTADOS MÉTODO}

El método de investigación utilizado fue el método descriptivo; ya que éste describe las situaciones y eventos, además mide diversos aspectos del fenómeno a investigar. El estudio descriptivo se desarrolla describiendo las situaciones y eventos, es decir cómo se manifiesta el fenómeno a investigar, ya que éste busca especificar las propiedades importantes del problema en cuestión. El método descriptivo mide independientemente los conceptos y también pueden ofrecer la posibilidad de predicciones aunque sean muy rudimentarias. (Sánchez y Reyes, 2002) (5).

\section{DISEÑO DE INVESTIGACIÓN}

Para la selección del diseño de investigación se ha utilizado como base el libro de Hernández, Fernández y Baptista (2010) (6), titulado "Metodología de la Investigación" Según estos autores el diseño adecuado para esta investigación es de tipo descriptivo correlacional.

Su diagrama representativo es el siguiente:

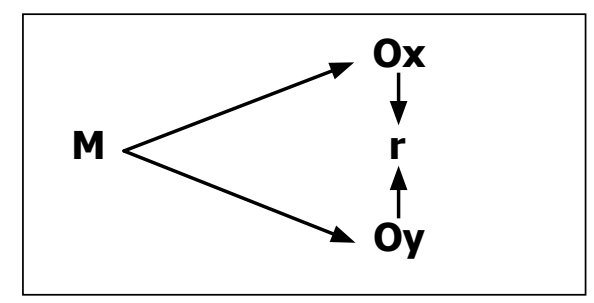

En el esquema:

$$
\begin{array}{ll}
\mathrm{M} & =\text { Muestra de investigación } \\
\mathrm{Ox}, \mathrm{Oy} & =\text { Observaciones de las variables } \\
\mathrm{r} & =\text { Relaciones entre variables }
\end{array}
$$

En este caso, se quiere correlacionar las variables competencias laborales y aprendizaje organizacional de los docentes de las instituciones educativas del distrito de Comas.

\section{MUESTRA}

La muestra de estudio que utilizamos, en tanto se ajusta a nuestras necesidades, es una muestra no probabilística de tipo intencionado en la medida que es el investigador quien ha determinado de manera intencional el lugar y la muestra con la que se trabajo. Esta decisión se tomo considerando que aplicar los instrumentos es una tarea muy complicada puesto que los docentes colaboran muy poco, sin embargo creemos que la muestra tomada es bastante representativa. 
Se tomo como muestra docentes de cinco instituciones educativas que en total suman 240 , con edades que fluctúan entre 23 y 57 años de edad presentando una predominancia los profesores cuyas edades están en los $30,32,36$ y 45 años de edad. También se aprecia que la muestra esta conformada mayoritariamente por mujeres $59.2 \%$, frente al $40.8 \%$ de los varones, igualmente de puede apreciar que los docentes provenientes de institutos pedagógicos son mayoritarios, $65.3 \%$ frente al 34.7 que proviene de las universidades.

Tabla 1. Composición general de la muestra

\begin{tabular}{ccc}
\hline Sexo & F & \% \\
\hline Masculino & 98 & 40.8 \\
Femenino & 142 & 59.2 \\
\hline $\begin{array}{c}\text { Institución } \\
\text { Educativa }\end{array}$ & \\
\hline $\mathbf{1}$ & 40 & 16.7 \\
\hline $\mathbf{2}$ & 46 & 19.2 \\
\hline $\mathbf{3}$ & 51 & 21.3 \\
\hline $\mathbf{4}$ & 50 & 20.8 \\
\hline $\mathbf{5}$ & 53 & 22.1 \\
\hline
\end{tabular}

\section{INSTRUMENTOS}

Los instrumentos utilizados en el desarrollo de la presente investigación, fueron:

El inventario de competencias laborales de Ana María Orti Gonzáles (2004) (7), adaptado para el trabajo con docentes por el autor de la presente investigación. Este instrumento esta compuesto por ocho escalas independientes (capacidad de riesgo, capacidad creativa, capacidad de organización, capacidad de comunicación, capacidad de liderazgo, capacidad de crear redes sociales, capacidad de detección de oportunidades y capacidad de trabajo en equipo) que evalúan las capacidades laborales que presentan los docentes en su actividad profesional.

Inventario de aprendizaje organizacional, elaborado por Delio Ignacio Castañeda (2007) (8), igualmente adaptado para el trabajo con docentes por el autor de la presente investigación. Este inventario esta compuesto por seis escalas independientes (aprendizaje individual, aprendizaje grupal, aprendizaje en la organización, cultura organizacional, transferencia de información y formación) que evalúan el aprendizaje organizacional que ocurre regularmente en una institución, tanto a nivel de adquisición y transferencia de la información como de la facilitación de la misma.

\section{ANÁLISIS DE VALIDEZ Y CONFIABILIDAD DE LOS INS- TRUMENTOS}

En la medida de que se necesita garantizar la idoneidad de los instrumentos de evaluación, se procedió a realizar los análisis estadísticos respectivos que nos indiquen sus niveles de validez y confiabilidad. El análisis psicométrico del inventario de competencias laborales indica que sus escalas alcanzan alfa de cronbach que fluctúan entre 0.88 y 0.96 por lo que se considera que la prueba es confiable

El análisis psicométrico del inventario de aprendizaje organizacional indica que sus escalas alcanzan alfa de cronbach que fluctúan entre 0.75 y 0.93 por lo que se considera que la prueba es confiable.

Los análisis de la Validez de los instrumentos, realizado a través del Análisis factorial Exploratorio nos muestran que las pruebas presentan validez de Constructo.

\section{RESULTADOS}

Tabla 2. Análisis de la Bondad de Ajuste de la Curva Normal de las Variables estudiada

\begin{tabular}{|l|c|c|}
\hline \multicolumn{1}{|c|}{ VARIABLE } & MEDIA & D.S. \\
\hline Capacidades Laborales & & \\
\hline Capacidad de Riesgo & 24.16 & 5.43 \\
\hline Capacidad creativa & 35.26 & 4.59 \\
\hline Capacidad de Organización & 42.65 & 6.04 \\
\hline Capacidad de comunicación & 44.00 & 5.84 \\
\hline Capacidad de Liderazgo & 43.78 & 5.91 \\
\hline Capacidad de crear redes sociales & 17.78 & 2.41 \\
\hline Capacidad de detección de oportunidades & 21.81 & 1.24 \\
\hline Capacidad de trabajo en equipo & 34.31 & 2.32 \\
\hline
\end{tabular}




\begin{tabular}{|l|l|l|l|}
\hline $\begin{array}{l}\text { Aprendizaje Organizacional } \\
\text { Aprendizaje individual }\end{array}$ & 14.28 & 2.84 & 2.29 \\
\hline Aprendizaje grupal & 20.70 & 3.44 & 2.22 \\
\hline Aprendizaje organizacional & 19.25 & 3.05 & 1.65 \\
\hline Cultura aprendizaje & 18.40 & 3.33 & 1.25 \\
\hline Transferencia información & 14.88 & 3.10 & 2.90 \\
\hline Formación & 18.45 & 3.87 & 1.95 \\
\hline
\end{tabular}

Los resultados del análisis exploratorio de los datos (ver tabla $N^{\circ} 2$ ), en lo que se refiere a la forma de distribución, efectuado a través del test de bondad de ajuste a la curva normal de Kolmogorov-Smirnov, indica que en todas las variables estudiadas, se ob- tienen coeficientes K-S Z que son estadísticamente significativos, por lo que se puede concluir que no se presentan adecuadas aproximaciones a la curva normal, por lo que es necesario utilizar para el análisis de los datos, estadísticas no paramétricas.

Tabla 3. Matriz de Correlaciones entre los Puntajes de las competencias laborales y las escalas del aprendizaje organizacional

\begin{tabular}{|c|c|c|c|c|c|c|c|}
\hline \multirow{2}{*}{$\begin{array}{c}\text { Competencias } \\
\text { Laborales }\end{array}$} & \multicolumn{7}{|c|}{ Aprendizaje Organizacional } \\
\hline & Indiv & Grupal & Org & Cultura & Infor & Form & Total AO \\
\hline Riesgo & $0.26^{\star *}$ & $0.40^{* * *}$ & $0.55^{\star * \star}$ & $0.51^{* * *}$ & 0.09 & $0.23^{*}$ & $0.45^{* * *}$ \\
\hline Creatividad & $0.19^{*}$ & $0.39^{* *}$ & $0.31^{* *}$ & $0.22^{*}$ & 0.09 & 0.03 & $0.29^{* *}$ \\
\hline Organización & 0.01 & $0.21^{*}$ & $0.20^{*}$ & $0.27^{* *}$ & -0.09 & -0.03 & $0.19^{*}$ \\
\hline comunicación & 0.01 & $0.18^{*}$ & 0.15 & 0.13 & -0.16 & -0.13 & 0.09 \\
\hline Liderazgo & 0.07 & $0.25^{\star *}$ & $0.19^{*}$ & $0.31^{* *}$ & 0.01 & 0.00 & $0.25^{\star *}$ \\
\hline Crear redes & 0.08 & 0.11 & 0.13 & 0.07 & 0.07 & 0.05 & $0.18^{*}$ \\
\hline Oportunidad & 0.12 & $0.18^{*}$ & 0.13 & $0.21^{*}$ & -0.06 & -0.06 & $0.18^{*}$ \\
\hline Trabajo en equipo & -0.04 & $0.20^{*}$ & 0.12 & $0.31^{* *}$ & -0.08 & 0.03 & $0.27^{* *}$ \\
\hline Total CL & 0.14 & $0.30^{* *}$ & $0.29^{* *}$ & $0.36^{* * *}$ & -0.04 & 0.03 & $0.29^{* *}$ \\
\hline
\end{tabular}

El análisis de las correlaciones entre las competencias laborales y el aprendizaje organizacional indica que existen correlaciones significativas y positivas en varios casos, resaltándose las correlaciones entre el total de ambas variables. Es necesario se- ñalar que estas correlaciones no son precisamente altas, seguramente porque entre nuestros docentes no esta claramente definido la importancia que estas variables tienen para el desarrollo de su trabajo y por lo tanto no le presta la atención debida. 
Tabla 4. Prueba $Z$ de comparación de medias de los puntajes de las Competencias laborales por sexo

\begin{tabular}{|l|c|c|c|c|c|}
\hline \multirow{2}{*}{ Variables } & \multicolumn{2}{|c|}{ Varón } & \multicolumn{2}{c|}{ Mujer } & \multirow{2}{*}{ N } \\
\cline { 2 - 5 } & $\mathbf{M}$ & D.E. & M & D.E & \\
\hline Riesgo & 23.38 & 4.38 & 25.30 & 6.54 & $-1.98^{*}$ \\
\hline Creatividad & 35.07 & 3.45 & 35.55 & 5.88 & -0.56 \\
\hline Organización & 42.15 & 5.33 & 43.36 & 6.93 & -1.08 \\
\hline comunicación & 44.12 & 5.51 & 43.81 & 6.35 & 0.28 \\
\hline Liderazgo & 43.74 & 5.35 & 43.83 & 6.70 & -0.08 \\
\hline Crear redes & 17.91 & 2.10 & 17.59 & 2.82 & 0.72 \\
\hline Oportunidad & 21.67 & 2.99 & 22.02 & 3.83 & -0.55 \\
\hline Trabajo en equipo & 34.18 & 4.35 & 34.51 & 5.54 & -0.36 \\
\hline Total CL & 262.25 & 23.87 & 266.00 & 40.25 & -0.63 \\
\hline
\end{tabular}

$\mathrm{p}<.05$

$\mathrm{n}=240$

El análisis comparativo de las Competencias laborales por sexo, presentado en la tabla $N^{\circ} 4$, realizado a través de la prueba $Z$ de diferencia de medias independientes, permite notar que existen diferen-

cias solo en la escala Capacidad de Riesgo, notándose que las mujeres presentan valores más altos que los varones, en los demás casos no existen diferencias.

Tabla 5. Prueba $Z$ de comparación de medias de los puntajes de las Escalas de Aprendizaje Organizacional por sexo

\begin{tabular}{|c|c|c|c|c|c|}
\hline \multirow{2}{*}{ Variables } & \multicolumn{2}{|c|}{$\begin{array}{l}\text { Varón } \\
\mathrm{N}=98\end{array}$} & \multicolumn{2}{|c|}{$\begin{array}{c}\text { Mujer } \\
\mathrm{N}=142\end{array}$} & \multirow{2}{*}{$\mathbf{Z}$} \\
\hline & M & D.E. & $\mathbf{M}$ & D.E & \\
\hline Aprendizaje individual & 13.73 & 3.26 & 15.08 & 1.84 & $-2.61^{*}$ \\
\hline Aprendizaje grupal & 20.29 & 3.66 & 21.28 & 3.02 & -1.55 \\
\hline Aprendizaje organizacional & 18.53 & 3.30 & 20.28 & 2.31 & $-3.20^{* *}$ \\
\hline Cultura aprendizaje & 17.43 & 3.32 & 19.79 & 2.85 & $-4.04^{* *}$ \\
\hline Transferencia información & 14.57 & 3.67 & 15.32 & 1.95 & -1.30 \\
\hline Formación & 17.60 & 4.35 & 19.67 & 2.64 & $-2.96^{*}$ \\
\hline Total Aprendizaje Org & 102.18 & 17.43 & 111.44 & 11.54 & $-3.25^{* *}$ \\
\hline
\end{tabular}


El análisis comparativo del aprendizaje organizacional por sexo (ver tabla $N^{\circ} 5$ ), efectuado a través de la prueba $Z$ de diferencia de medias independientes, permite notar que existen diferencias significativas en los casos de Aprendizaje individual, Aprendizaje organizacional, Cultura aprendizaje, Formación y el total del Aprendizaje Organizacio- nal, notándose que en todos los casos las docentes mujeres superan a los docentes varones, es decir, las mujeres presente una mejor disposición para el aprendizaje en las organizaciones, probablemente porque sienten la necesidad de mostrarse más competentes y mejor dispuestas para el trabajo que los varones.

Tabla 6. Análisis descriptivos de las Competencias laborales

\begin{tabular}{|c|c|c|c|c|}
\hline \multicolumn{2}{|c|}{ Respuestas } & Alto & Medio & Bajo \\
\hline \multirow{2}{*}{ Capacidad de Riesgo } & Casos & 108 & 112 & 20 \\
\cline { 2 - 5 } & $\%$ & 45.0 & 46.7 & 8.3 \\
\hline \multirow{2}{*}{ Capacidad creativa } & Casos & 220 & 20 & 0 \\
\cline { 2 - 5 } Capacidad de Organización & $\%$ & 91.7 & 8.3 & 0.0 \\
\cline { 2 - 5 } & Casos & 208 & 32 & 0 \\
\hline \multirow{2}{*}{ Capacidad de comunicación } & Casos & 86.7 & 13.3 & 0.0 \\
\cline { 2 - 5 } & $\%$ & 228 & 12 & 0 \\
\hline \multirow{2}{*}{ Capacidad de Liderazgo } & Casos & 212 & 28 & 0.0 \\
\cline { 2 - 5 } Capacidad de crear redes & $\%$ & 88.3 & 11.7 & 0.0 \\
\cline { 2 - 5 } & Casos & 212 & 28 & 0 \\
\hline Capacidad de & $\%$ & 88.3 & 11.7 & 0.0 \\
\cline { 2 - 5 } & Casos & 188 & 52 & 0 \\
\hline \multirow{2}{*}{ Capacidad de equipo } & $\%$ & 78.3 & 21.7 & 0.0 \\
\cline { 2 - 5 } & Casos & 212 & 11.7 & 0.0 \\
\hline
\end{tabular}

$N=240$

Los resultados obtenidos en la Tabla $N^{\circ} 6$ nos indican que los profesores de la muestra valoran bastante bien sus competencias laborales, puesto que los más altos puntajes se encuentran entre los niveles altos y medios.

Tabla 7. Análisis descriptivos del Aprendizaje Organizacional

\begin{tabular}{|c|c|c|c|c|}
\hline \multicolumn{2}{|c|}{ Respuestas } & Alto & Medio & Bajo \\
\hline \multirow{3}{*}{ Aprendizaje individual } & Casos & 92 & 112 & 16 \\
\cline { 2 - 5 } & $\%$ & 38.3 & 55.0 & 6.7 \\
\hline \multirow{3}{*}{ Aprendizaje grupal } & Casos & 188 & 44 & 8 \\
\cline { 2 - 5 } Aprendizaje organizacional & $\%$ & 78.3 & 18.3 & 3.3 \\
\cline { 2 - 5 } & Casos & 128 & 104 & 8 \\
\hline \multirow{3}{*}{ Cultura aprendizaje } & $\%$ & 53.3 & 43.3 & 8 \\
\cline { 2 - 5 } Transferencia información & Casos & 96 & 56.7 & 3.3 \\
\cline { 2 - 5 } & Casos & 40.0 & 92 & 28 \\
\hline Formación & $\%$ & 120 & 38.3 & 11.7 \\
\cline { 2 - 5 } & Casos & 50.0 & 124 & 8 \\
\hline
\end{tabular}


Los resultados obtenidos en la Tabla $N^{\circ} 7$ nos indican que los profesores de la muestra valoran medianamente bien la capacidad de aprendizaje organizacional existente en sus instituciones educativas. Los resultados muestran que los más altos puntajes se encuentran básicamente en los niveles medios.

\section{ANÁLISIS Y DISCUSIÓN}

Los análisis psicométricos a los que fue sometido el inventario de competencias laborales docente, revelan que los 62 reactivos que conforman el inventario en las escalas asignadas por la autora de la versión original deben permanecer tal cual fueron elaboradas y asignadas a cada escala. Asimismo, los coeficientes Alfa de Cronbach alcanzados en las ocho escalas oscilan entre 0.88 y 0.96 y prueba total alcanza el 0.92 , lo cual indica que el instrumento es confiable.

Por otro lado los análisis psicométricos a los que fue sometido el Inventario de aprendizaje organizacional, revelan que todos los ítems forman parte de dicho inventario y corresponden a cada una de las seis escalas asignadas previamente. El instrumento es confiable en la medida que sus escalas obtuvieron coeficientes Alfa de Cronbach con valores que oscilan entre 0.75 y 0.93 , obteniendo además un alfa de Cronbach total para toda la prueba de 0.89 .

En ambos casos los resultados del Análisis Factorial Exploratorio indica que las pruebas alcanzan valores suficientes como para afirmar que presentan validez de constructo, por lo que su uso y resultados quedan plenamente garantizados.

En lo que se refiere a los resultados de la investigación alcanzados, estos nos indican la existencia de correlaciones significativas entre las variables en estudio, lo cual es un indicador de que ambas variables son realmente importantes en el funcionamiento de una institución educativa, lo cual confirmaría las formulaciones teóricas desarrolladas en el marco teórico, las mismas que afirman la importancia de ligar permanentemente estos dos aspectos en toda institución que pretende alcanzar altos niveles de calidad. Este hecho tiene su explicación en el sentido de que la competencia laboral es el producto del dominio de conceptos, destrezas y actitudes; ser competente significa que la persona tiene el conocimiento declarativo (la información y conceptos), es decir, sabe lo que hace, por qué lo hace y conoce el objeto sobre el que actúa. Ser competente también implica, tener la capacidad de ejecución, es decir el conocimiento procesal o las destrezas intelectuales y psicomotoras para en efecto llevar a cabo la ejecución sobre el objeto. Finalmente ser competente implica tener la actitud o disposición (conocimiento actitudinal) para querer hacer uso del conocimiento declarativo y procesal y actuar de manera que se considera correcta, (Villarini, 1996) (9). Como se puede observar las relaciones con el aprendizaje organizacional son evidentes toda vez que el conocimiento en las organizaciones proviene de los individuos y van hacia la organización.

Los análisis de las diferencias entre los docentes varones y mujeres respecto de las variables competencias laborales y aprendizaje organizacional, nos muestran que las mujeres presentan valores superiores a los varones, particularmente en lo que respecta al aprendizaje organizacional. Pareciera ser que las mujeres muestran una mejor disposición no solo para el aprendizaje, sino también para el trabajo, tal vez por la necesidad de mostrar que son iguales o más competentes que los varones por lo que merecen tener las mismas oportunidades laborales que los varones.

Finalmente me parece necesario insistir sobre la importancia del aprendizaje organizacional el mismo que se entiende en primera instancia como el aprendizaje que ocurre dentro de las organizaciones, como el proceso mediante el cual las personas de la organización, intercambian con sus propias características, acciones y experiencias, la cultura acumulada, tanto en lo que se refiere a conocimientos (conceptuales, instrumentales y operacionales), como habilidades, emociones, sentimientos, valores y generan los suyos propios, de tal manera que todas las personas de la organización, sin excepción, lo puedan utilizar, en los procesos de su actividad cotidiana, que puede ser una actividad individual, grupal, colectiva, o social.

\section{CONCLUSIONES}

1. Los análisis estadísticos realizados demuestran la existencia de relaciones significativas y positivas entre el total de las competencias laborales y el total del Aprendizaje Organizacional de los docentes de las Instituciones Educativas del Distrito de Comas.

2. El análisis comparativo de las competencias laborales docente por Sexo, indica que existen diferencias estadísticas significativas solo en el caso Capacidad de Riesgo notándose que las mujeres presentan valores más altos que los varones.

3. El análisis comparativo de la Prueba de Aprendizaje Organizacional por Sexo, indica que existen diferencias estadísticas significativas en los 
casos de Aprendizaje individual, Aprendizaje organizacional, Cultura de aprendizaje, Formación y el Total del Aprendizaje Organizacional notándose que en todos los casos las docentes mujeres superan a los docentes varones.

4. Los análisis descriptivos muestran que en el caso de las competencias laborales, los niveles son relativamente altos, luego sigue el nivel medio y en último lugar los niveles bajos.

5. Los análisis descriptivos muestran que en el caso del Aprendizaje Organizacional, los niveles son básicamente medios, luego sigue el nivel alto y en último lugar los niveles bajos.

\section{REFERENCIAS BIBLIOGRÁFICAS}

[1] Drucker, P (1993). La sociedad post capitalista. Barcelona: Grupo Editorial Norma.

[2] Pawlowsky, P. (2001). The Treatment of Organizational Learning in Management Science. En Dierkes, M., Berthoin-Antal, A., Child, J. \& Nonaka, I (Eds.). Handbook of Organizational Learning and Knowledge. Nueva York: Oxford University Press.

[3] Argyris, C. (2002): Sobre las organizaciones que aprenden. México: Oxford.
[4] Valdés Veloz, H. (2009) Manual de buenas prácticas de evaluación del desempeño profesional de los docentes. Consejo Nacional de Educación. Lima.

[5] Sánchez y Reyes, (2002). Metodología y diseños en la Investigación Científica. Lima. Universidad Ricardo Palma.

[6] Hernández, Fernández y Baptista (2010) Metodología de la Investigación. México. Ed Mc Graw Hill.

[7] Orti Gonzalez, A. (2004). Desarrollo de una Escala para la Definición de las Dimensiones de Autoeficacia Percibida Emprendedora. III Congreso de Metodología de Encuestas. Congreso de Metodología de Encuestas. Num. 3. Granada. Universidad de Granada.

[8] Castañeda, D.; (2007). Validación de una escala de niveles y condiciones de aprendizaje organizacional. Univ. Psychol., Bogotá, v. 6, n. 2, ago. 2007 . Disponible en <http://pepsic.bvsalud. org/scielo.php?script=sci_arttext\&pid=S165792672007000200004\&lng=pt\&nrm=iso>.

[9] Villarini, (1996). Capacitación de Competencias. México: Editorial Limusa 